\section{Motivating the adoption of new community-minded behaviors: An empirical test in Nigeria}

\author{
Graeme Blair $^{1 *{ }^{\dagger}}$, Rebecca Littman ${ }^{2 *}{ }^{\dagger}$, Elizabeth Levy Paluck ${ }^{3,4 * \dagger}$
}

\begin{abstract}
Social scientists have long sought to explain why people donate resources for the good of a community. Less attention has been paid to the difficult task of motivating the first adopters of these important behaviors. In a field experiment in Nigeria, we tested two campaigns that encouraged people to try reporting corruption by text message. Psychological theories about how to shift perceived norms and how to reduce barriers to action drove the design of each campaign. The first, a film featuring actors reporting corruption, and the second, a mass text message reducing the effort required to report, caused a total of 1181 people in 106 communities to text, including 241 people who sent concrete corruption reports. Psychological theories of social norms and behavior change can illuminate the early stages of the evolution of cooperation and collective action, when adoption is still relatively rare.
\end{abstract}

\section{INTRODUCTION}

Why do people donate time, money, or other resources to their communities? Scientists view this question about community-minded behavior as part of a broader question about why humans cooperate, which sets apart large-scale human behavior from the behavior of other species (1). Research has uncovered several factors that drive and constrain community-minded behavior, including expectations of paybacks (2) and of punishments for free riders (3), internalized decision-making tendencies that favor cooperation over selfishness (4), and perceived social norms (5). A rich tradition of research on the evolution of collective action for public goods provision was inspired by the pioneering work of Ostrom (6), but few studies in this tradition focus on how community-minded behaviors are initially adopted. Communityminded behaviors that are rare (because they are novel or, for whatever reason, are not widely adopted) may carry special costs and risks. These costs and risks accrue from the fact that people have difficulty choosing and acting on alternatives to the status quo $(7,8)$, people's contributions may not be matched by other community members without coordinated punishment of free riders $(3,9)$, and people may perceive social, legal, or safety risks associated with being one of few to participate. Understanding why people would sacrifice resources for their community, particularly when it is not socially recognized as a common or desirable behavior, is important for understanding the full evolution of a collective action strategy $(6,10)$.

Learning how community-minded contributions are adopted and become normative is also pragmatically important because voluntary community contributions are often encouraged in contexts where government or private sector initiatives are infeasible or unsuccessful. For example, reporting social or political problems via text message is a recently invented solution used by a growing number of political activists, civil society organizations, and technology experts (11). Initiatives in countries like Kenya, Haiti, and India have asked people to contribute information about local violence, natural disasters, or corruption to help

\footnotetext{
${ }^{1}$ Department of Political Science, UCLA, Los Angeles, CA 90095, USA. ${ }^{2}$ Sloan School of Management, Massachusetts Institute of Technology, Cambridge, MA 02139, USA. ${ }^{3}$ Department of Psychology, Princeton University, Princeton, NJ 08544, USA. ${ }^{4}$ Woodrow Wilson School of Public and International Affairs, Princeton University, Princeton, NJ 08544, USA.

*Corresponding author. Email: graeme.blair@ucla.edu (G.B.); rebecca.littman@mit. edu (R.L.); epaluck@princeton.edu (E.L.P.)

tThese authors contributed equally to this work.
}

other citizens navigate crises and to identify areas in need $(12,13)$. Studies of technology adoption show that once a certain threshold of new users is achieved, bandwagon effects, herding, and information cascades can spur new behaviors to become widespread in a society $(14,15)$. However, the success of these initiatives depends, as a first step, on persuading an initial group of people to try a behavior that is outside of the status quo.

The current study addresses this first step, of how to persuade an initial group of people to try a new form of community-minded action. We identify two major barriers to adopting new actions: the perception that no one else will join (a problem of social norms) and minor logistical or technical barriers (a problem of personal and structural capacity). Psychological theories of social norms and behavior change offer ideas for addressing each barrier-specifically, mechanisms for influencing individuals' perceptions of social norms, and small interventions for increasing individuals' personal capacity, given structural and cognitive constraints.

Psychological theory defines a perception of a social norm as an individual's perception of how widespread or desirable a behavior is or is becoming within the community $(16,17)$. Research suggests that norm perception is a strong motivator of human behavior (18). For motivating rare behaviors, perceptions of a norm can be influenced even before changing the actual rate of the behavior in a community (19). Studies suggest that this is possible in part because people overgeneralize from the public behavior of role models to their perception of the overall community norm $(20,21)$. Role models who influence norm perceptions can be people in one's community who are high status $(22,23)$, who receive lots of attention (20), and, in some cases, who break with tradition (24). However, people can even use fictional characters as role models, such as characters in a soap opera (25). Thus, one mechanism for changing norm perceptions is to highlight the behavior of role models, real or fictional (19).

Psychological theory on behavior change also highlights the importance of removing minor but psychologically important barriers to a behavior, using a "nudge" (26). A nudge-such as a reminder (27) or a new default (28)-helps a person to accomplish a behavior toward which he or she is positively inclined by removing barriers to the behavior. While this insight may seem intuitive, behavioral research has demonstrated that what may seem like a materially insignificant cost, such as memorizing a number or filling out a form, can actually be a psychologically large barrier given constraints on individual cognitive or 
motivational capacity (26). A wealth of studies show that using nudges to remove minor barriers significantly increases rates of consequential behaviors like showing up to a court date (29), successfully enrolling in college (30), and voting (31).

Thus far, psychological theories about norms and behavior change have been successfully applied in Western democracies to improve low rates of community-minded behaviors. Using a large-scale field experiment, we evaluate these two psychological theories in a non-Western context where activists sought to encourage the rare behavior of corruption reporting. In Nigeria, reporting corruption to nongovernmental agencies is part of an envisioned citizen-driven campaign to reduce the country's endemic corruption (32). Activist groups hope that large numbers of people will report their experiences with corruption, which would be used to write advocacy reports testifying to the widespread nature and negative effects of corruption on the population. However, citizen corruption reporting is extremely uncommon in Nigeria. Previous efforts to encourage reporting have been uniformly unsuccessful, regardless of the recipient of the reporting and regardless of the technology used. Nationwide campaigns via phone, text message, and the internet have elicited no more than 140 reports per year, out of a population of 174 million $(33,34)$.

The failure of previous corruption-reporting campaigns in Nigeria cannot be attributed to a lack of concern about the issue, since Nigerians consistently name corruption as one of the top problems facing their country (35). Our pilot research in Nigeria, including a survey of 345 randomly sampled individuals in the four states where our study took place, indicated that just under $80 \%$ of citizens believe the police, civil servants, and their state governments were corrupt. Eighty-three percent reported being angry they had to pay bribes, and $60 \%$ reported being very angry. Our research also suggested the importance of the two barriers to reporting. With respect to perceived norms of reporting, respondents indicated that corruption reporting is rare; with respect to logistical barriers, they reported that there are no means to report. Related to both assertions, $30 \%$ of pilot respondents said that they felt reporting corruption was useless, and 50\% said reporting was dangerous.

In the present experiment, we tested a norms intervention, which aimed to address individuals' perception that corruption reporting is rare, and a nudge intervention, which aimed to minimize the perceived logistical barriers to corruption reporting. We first worked with a Nigerian anticorruption group to establish a toll-free text messaging platform. We then launched two campaigns that raised awareness of the platform and that attempted to (i) shift perceptions of the norms of corruption reporting and (ii) reduce barriers to reporting using a nudge. We tracked corruption reports texted into the platform from each community for 160 days. We also fielded a survey in each community several days after both campaigns were implemented.

The first campaign was a 2-hour Nigerian ("Nollywood") feature film produced for this project with well-known actors. It was filmed in the Niger Delta region where the study took place. The film's dramatic storyline incorporated corruption as part of the characters' everyday lives. The film campaign was rolled out in a between-community design. The design entailed that half of the study communities received a treatment version of the film and half received a placebo version. The packaging of the two versions was identical except that different phone numbers for texting reports were included on the covers in small letters. The treatment version included scenes in which the actors reported corruption using our texting platform as part of the overall corruption storyline (Fig. 1, A and B). These scenes showing role models reporting corruption were designed to shift viewers' perceptions of corruption-reporting norms. Specifically, the role models' behavior was used to increase audience perception that other Nigerians report and approve of corruption reporting. The role models in the film were fictional but also high-status actors filmed in the audience's local Nigerian context. A placebo version of the film cut these corruptionreporting scenes, which were not central to the film's resolution.

Both the treatment and placebo versions of the film advertised the corruption-reporting platform at the beginning, middle, and end of the film with a banner across the screen and on the film packaging. Thus, all film viewers had the information needed to use the corruption-reporting platform. Because we randomized which version of the film was distributed in each community, we are able to estimate the causal effect of the role models in the treatment film compared with the placebo on corruption-reporting and perceived norms.

The second campaign was a mass text message sent to all customers of Nigeria's largest mobile phone provider in each study community, after the community received either the treatment or placebo film. The mass text was sent to people in each community on a randomly assigned date a few weeks following the film distribution using a stepped wedge design. By virtue of this randomization, we are able to estimate the causal effect of the message by comparing response rates before and after the randomly assigned date of the nudge. The message alerted individuals to the corruption-reporting platform and explained the ease of reporting (Fig. 2A). In this way, the message was designed to reduce barriers to reporting by clearly explaining the reporting campaign, allowing recipients to simply hit "reply" to report, and emphasizing that correspondence with the corruption platform was free (an unusual feature; see Methods and Materials for more details on each campaign).

Thus, the film campaign and the text message campaign both attempted to encourage individuals to adopt corruption reporting via text, either by promoting the perception that corruption reporting was becoming more normative, i.e., widespread and accepted (via role models in the film), or by reducing barriers to reporting (via a text-based reminder with simple and encouraging instructions). Neither campaign promised investigative action in response to a report, and any person who inquired via text message was told that reports would be used by a Nigerian activist organization. Both campaigns made salient the issue of corruption, although daily life already highlights this issue to the majority of Nigerians. Our film intervention controls for salience of the corruption-reporting platform: We compare corruption reports and norms among people who watched either the treatment or the placebo film, both of which provided information about the corruptionreporting campaign hotline. Within this film experiment, we assess whether corruption reporting is further boosted by a nudge from a text message sent on a randomly assigned day to both communities that received the treatment film or the placebo film.

\section{RESULTS}

Over the course of our 7-month study in 106 communities across the Niger Delta region in southeastern Nigeria (see figs. S1 and S2), the corruption-reporting platform received 3316 messages from 1685 unique senders. In total, 1181 unique senders texted in messages discussing corruption or the study's campaigns, including 241 unique individuals who sent a concrete corruption report that made explicit mention of a corrupt act, person, or institution (Fig. 3). As a benchmark, our 7-month study in 106 small southern Nigerian communities (for example, in urban neighborhoods as small as a quarter square mile) produced 1.7 times as many concrete corruption reports as 1 year of 
A

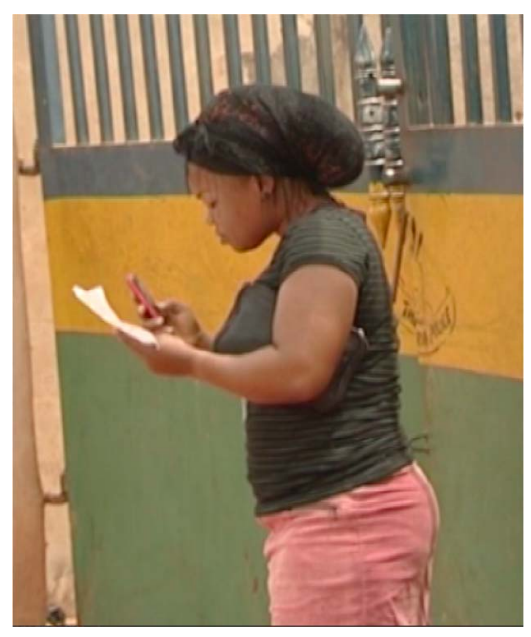

B

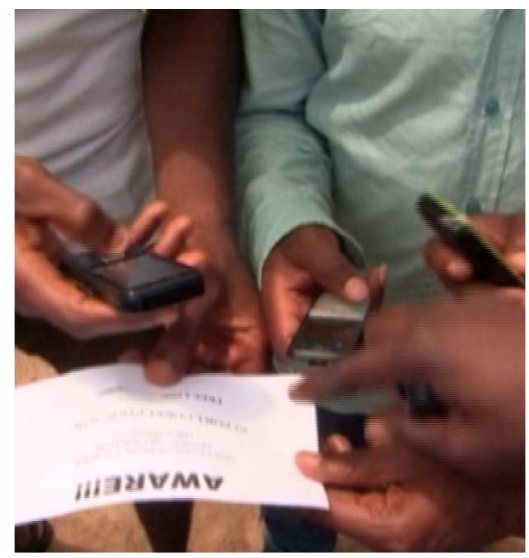

C

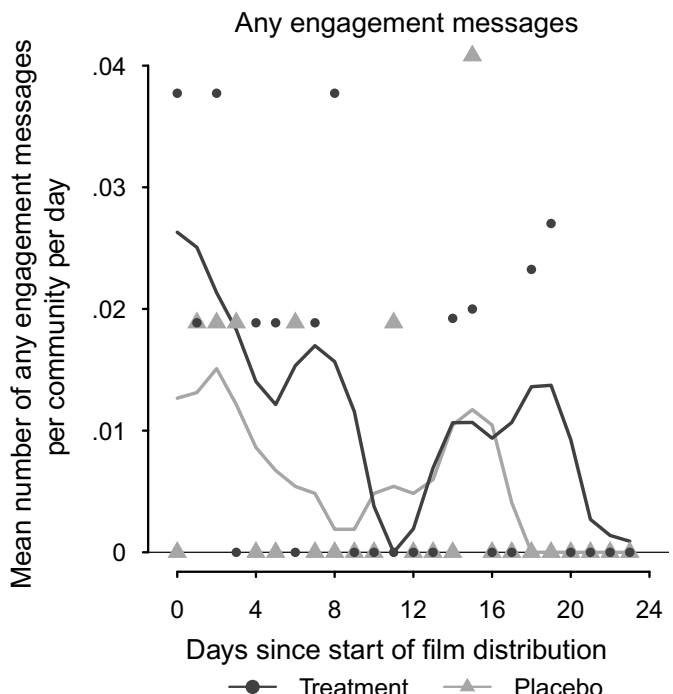

D

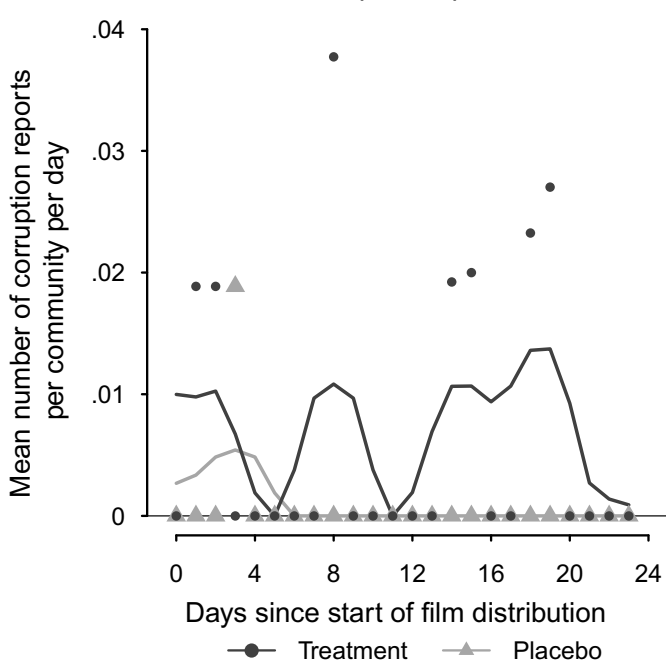

Fig. 1. Social norms campaign film scene and results. (A and B) Scenes from the treatment version of the Nollywood film, depicting characters texting in corruption reports. (Photo credit: Graeme Blair, University of California, Los Angeles; Rebecca Littman, Massachusetts Institute of Technology; Elizabeth Levy Paluck, Princeton University). (C and D) Time series plotting the average daily number of text messages that engaged with the campaign and that reported a concrete corruption event, respectively, from communities where we delivered the treatment versus placebo version of the film. Locally estimated scatterplot smoothing (LOESS) lines are overlaid.

the previous nationwide corruption-reporting campaign. People most commonly reported bribes and embezzlement perpetrated by politicians, law enforcement, and those in the education sector. Of the corruption-related messages, $86 \%$ arrived within the first 30 days after the campaigns began.

What caused people to report? A total of 31,800 copies of the treatment and placebo films were distributed to the 106 randomly sampled communities in a random order. A randomly assigned half of communities received the treatment film, and the other half the placebo version. Figure $1(\mathrm{C}$ and $\mathrm{D})$ shows that we received messages from communities where we distributed the treatment version of the film, but the average effect is close to zero from the placebo communities. We estimate that in the 15- to 34-day period between the start of film distribution and the launch of the mass text message, the treatment film caused an increase of 0.007 corruption reports per day in each community compared with the placebo film [confidence interval (CI), 0 to 0.013 ].

In each community, the mass text message was sent on a randomly assigned day 10 to 20 days after film distribution. On the day the mass text message arrived, we observed an estimated increase of 5.053 corruption-related messages in each community (CI, 4.06 to 6.047), including an increase of 0.91 concrete corruption reports (CI, 0.597 to 1.224; Fig. 2, B and C). Afterward, we observed a steep decay such that 3 days later, the effect of the campaign was 0.208 corruptionrelated messages per day in each community (CI, 0.038 to 0.378 ). The 
A

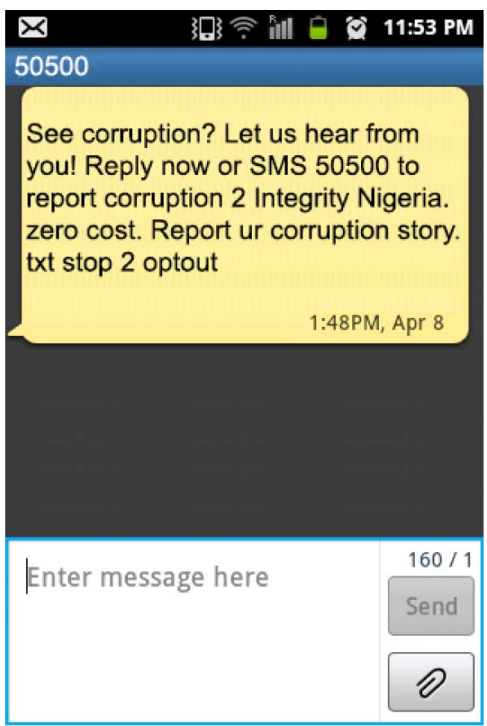

B

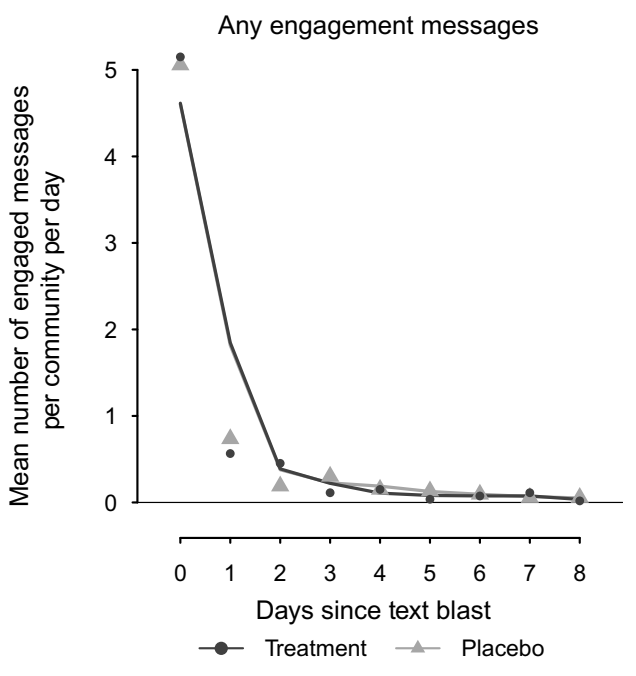

C

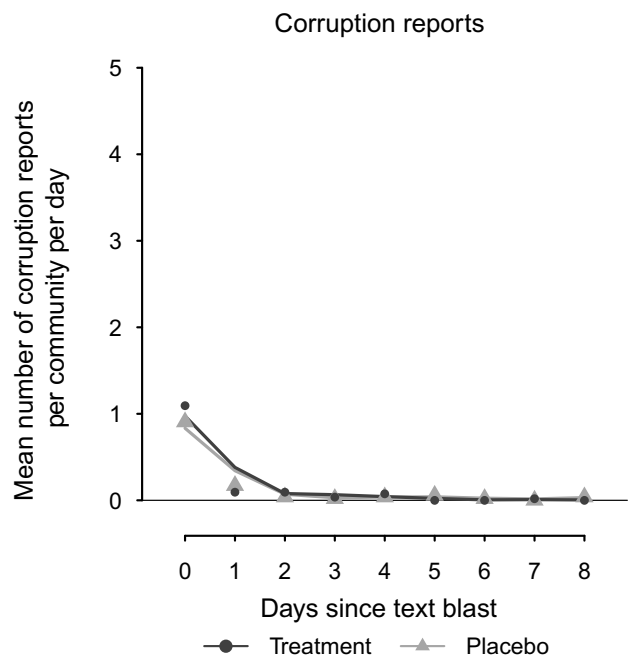

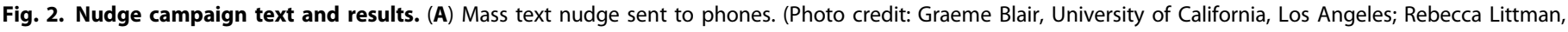

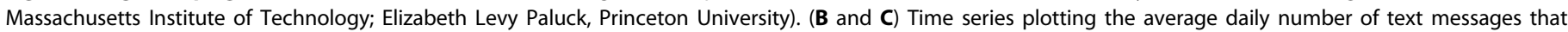

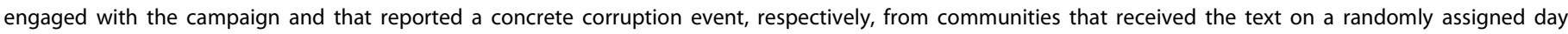
following the film distribution. LOESS lines are overlaid.

effects of the mass text message were not different in the placebo versus treatment film communities (difference, -0.009 ; CI, -0.03 to 0.012).

To test whether the treatment version of the film shifted viewers' perceptions of social norms, we surveyed 1399 randomly sampled film viewers from our study population (see the Supplementary Materials). We found that watching the film's characters text about corruption shifted the viewers' perceptions of norms in their community, specifically that corruption, and anger about corruption, was widespread (corruption $=0.083$ and CI, 0.025 to 0.142 ; anger $=0.116$ and CI, 0.035 to 0.197 ; fig. S4). Respondents who watched the treatment film also believed that their community viewed corruption reporting as dangerous to a significantly greater extent than the placebo film audience $(0.097$; CI, 0.016 to 0.178 ). But contrary to our prediction that viewers would overgeneralize the behavior of the characters in the film to others in their community, the treatment film did not increase perceptions of how widespread corruption reporting was or was becoming in their community, compared with viewers in placebo communities $(-0.003 ; \mathrm{CI},-0.052$ to 0.046$)$. One reason may be that the text reporting was not a visible behavior in their community: Only $18 \%$ of all respondents knew someone who planned to text a corruption report, and only $5 \%$ knew someone who actually had texted a corruption report.

\section{DISCUSSION}

Two campaigns in 106 communities, involving 17 additional minutes in a Nollywood film and one unsolicited mass text message, prompted 241 corruption reports and hundreds more text messages signaling interest in addressing corruption. The influence of each campaign operated independently: Communities receiving the treatment film were just as responsive to the mass text campaign as those that received the placebo film. While it is impossible to report a precise response rate given that we do not know how many people actually read the mass text or watched the film, we estimate a range from 0.002 (approximately 1 of
605 people sending a message) to 0.007 (approximately 1 of 145 people sending a message). The rate of 0.002 was calculated assuming that all 683,000 recipients received the mass text message and all 31,800 films were viewed; the rate of 0.007 was calculated assuming approximately one-quarter of the text messages were received on the basis of a phone audit that we conducted (see the Supplementary Materials) and that one-half of the films were viewed. Our campaigns elicited a much higher response rate than previous corruption-reporting campaigns in Nigeria, which received fewer than 140 messages from the entire country over the course of 1 year $(33,34)$. The response rate in our study is also substantially higher than that of a radio ad campaign in Uganda encouraging citizens to send messages to their Member of Parliament [estimated response rate $=0.0002$ over a 6-month period (36)]. Last, while our effects are statistically and substantively significant, they are smaller than the effects found in recent studies using a text or film campaign to target highly motivated citizens or behaviors that are driven by more immediate self-interest $(37,38)$.

The film campaign likely influenced corruption reporting through a mechanism other than shifting perceived norms about the commonality or desirability of reporting itself. Instead, the campaign may have caused reporting through the observed shift in perceived norms regarding the typicality of corruption and community members' feelings of anger about corruption. Previous work has shown the power of mobilizing collective emotions like anger to increase collective action (39). This mechanism may have encouraged more corruption reporting, despite the concurrent impression promoted by the treatment film that community members view corruption reporting as dangerous.

Encouraging people to try a community-minded action for the first time is a task separate from encouraging a long-term pattern of this behavior $(6,10)$. Our data cannot shed light on whether repeated mass text messages and greater media saturation of role models from popular culture would sustain the first month's rate of corruption reporting. Potentially, repeating the text messages and inserting more role models into mass media could successfully shift actual rates of corruption 


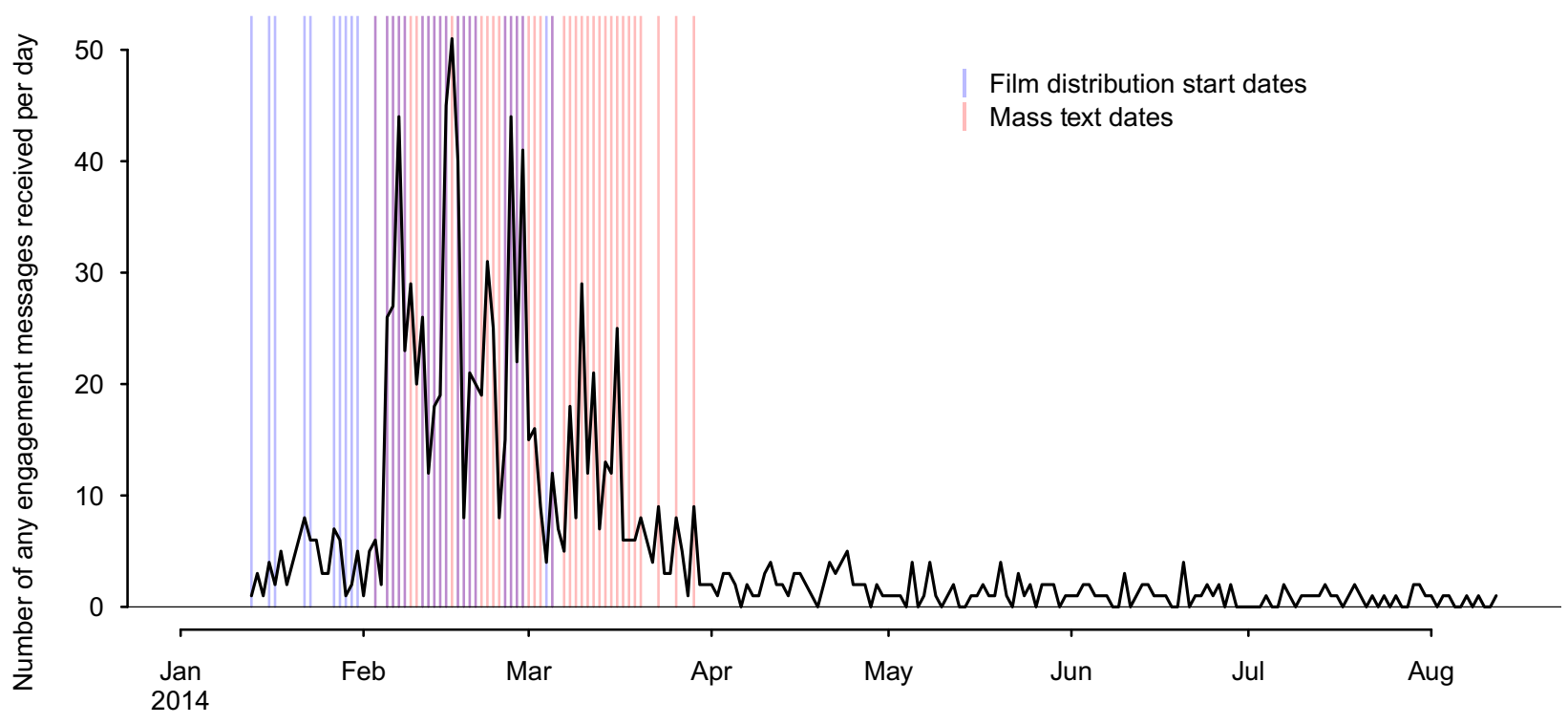

Fig. 3. Total number of text messages received per day from January 2014 to August 2014. The number of messages received per day is reported for Any Engagement messages that mentioned corruption or the two media campaigns, including Corruption Report messages that made explicit mention of a corrupt act, person, or institution.

reporting, in addition to perceived norms of corruption reporting. Interventions building on the present work could also advertise the existence of early adopters and early increases in corruption reporting. To understand how this new form of community-minded behavior might become an established convention, it would also be relevant to test whether publication of the received corruption reports would succeed in reducing actual levels of corruption, as a community-level payoff. These and other inquiries can contribute to understanding the adoption of rare community-minded behaviors and the evolution of cooperation and collective action.

\section{MATERIALS AND METHODS Identification of study population}

We estimated the coverage area of all mobile phone towers in four states of the Niger Delta (Akwa Ibom, Bayelsa, Delta, and Rivers) using their geographic coordinates. We then conducted a restricted random sampling of towers, such that communities were sufficiently separated from each other to avoid spillover of the mass text message and the Nollywood film. To do this, we simulated many samples of $215 \mathrm{com}$ munities meeting the distance restrictions and randomly selected one of those samples. Following a technical error with our mass text messaging service, we conducted the study on the unaffected subset of $106 \mathrm{com}$ munities within that representative sample (see the Supplementary Materials for further details). We also conducted a pilot study of our baseline survey that helped us to estimate rates of mobile phone usage, media consumption, corruption, and corruption reporting.

\section{Baseline survey}

We conducted a baseline survey in all 106 communities to gather data used to block communities for treatment assignment (see table S5). The survey was portrayed to participants as an independent survey from Princeton University. The sample for our baseline survey in each community was a randomly selected group of self-identified Nollywood film watchers (those who would likely purchase the Nollywood treatment film). In $88 \%$ of all communities, we interviewed between
12 and 20 people in the community; because of rigorous quality checks, 11 communities retained fewer than 12 participants (a minimum of 8). The number of surveys completed was balanced across treatment conditions (average $=15$ completed surveys under the placebo condition and 16 under the treatment condition). The survey measured a range of norms about corruption and corruption reporting. Surveyors also confirmed that the 106 communities had a video CD film seller (one of our treatment delivery methods) and reception from our mobile phone company partner.

\section{Treatment campaigns}

We developed two campaigns: a feature-length film with treatment and placebo versions, and a mass text message. The film was produced in collaboration with iROKO Partners, the leading distributor of films from the Nigerian film industry, known as Nollywood. Nollywood is the third largest film industry in the world, following Hollywood and Bollywood. The film produced for this project, titled Water of Gold, runs approximately 2 hours and stars well-known Nollywood actors. It tells the story of a poor fisherman named Natufe living in the Niger Delta and of his rags-to-riches brother, Priye. The two are close until Priye leaves their village, eventually returning many years later as a rich businessman. Against his brother's protests, Priye enters politics and begins to work with corrupt local politicians. The film depicts corruption harming Natufe and his neighbors' livelihoods, including bad medical care, police harassment, and oil companies disrespecting environmental concerns. Natufe becomes outspoken against the corrupt system in which they live.

In the treatment version of the film, Natufe and a local activist set up a toll-free "short-code" telephone number, and in additional scenes totaling $17 \mathrm{~min}$, they encourage community members to use the short code to report on corruption by text message. They explain that their activist group will use the text messages to write a public report. Subsequent treatment scenes separately depict men and women of varying social classes reporting corruption to the short code. These treatment scenes do not appear in the placebo film, which is otherwise identical. In both versions of the film, an information banner echoing the content 
of the mass text message appears on the screen four times: "See corruption? Let us hear from you! SMS 50500 to report corruption to Integrity Nigeria. Tell us your story. Text for FREE. Your number kept secret."

The mass text message treatment (see Fig. 2A) was received by all subscribers to the major mobile phone company within the treated phone tower's coverage area. A pilot survey that we conducted before the experiment revealed that $87 \%$ of people in the Niger Delta region with phones subscribed to that phone company ( $88 \%$ of all respondents reported owning a phone).

\section{Treatment assignment}

After we blocked communities into pairs using covariates measured in the baseline survey and characteristics of the community such as the number of mobile phone subscribers, we randomly assigned communities within each pair to receive either the treatment or the placebo film ( $n=53$ communities in each group). Next, we randomized the order in which films were distributed in communities. The order was randomized first among the pairs of communities and then between the treatment and placebo communities within each pair. Last, we randomized the timing of the mass text message for each blocked pair of communities, such that it was sent on a randomly assigned day within 14 to 20 days following the last day that films were distributed in a given community. (The actual time interval ranged from 10 to 20 days because of an administrative error in six communities.) This range was selected to give people in each community a chance to watch the film before receiving the mass text message.

\section{Film distribution}

Films were distributed in each community after the baseline survey, from 13 January 2014 to 9 March 2014. They were distributed by members of a Nigerian research firm who were trained on distribution protocols by the research team and were not part of the baseline survey enumeration team. Film distributors visited the main film seller in each community and introduced themselves as representatives of our film production partner. The distributors explained that they were promoting a new, unreleased film. As part of the promotion, they were there to give out two free copies of Water of Gold to customers who bought any Nigerian film from the film seller (one copy for the customer and one for the customer to give to a friend). In return for entry in a lottery for a Blackberry phone, customers who received the films were asked to provide their name and contact number, as well as the names and numbers of two friends with whom they usually watch Nigerian films. (The lottery was conducted and the phone delivered after the conclusion of the study.) These names and numbers were later used to randomly sample people to participate in the endline survey. To augment the number of films given out in such a short time, film distributors gave out an additional 100 free films to arbitrary people aged 18 and over in return for contact details. The distribution of 300 films in each community typically lasted from 3 to 5 days. All films were distributed by a member of our trained film distribution team who sat in the shop all day, not by the shop's film seller. Across the 106 communities in the sample, we distributed 31,800 films.

\section{Mass text message}

The mass text message was sent to all subscribers of the largest mobile phone provider in our study communities, as well as to the film buyers and their friends, whose numbers were collected by the film distribution team. Text messages were sent to 683,000 people across the 106 com- munities from 3 February 2014 to 29 March 2014. While we knew the exact number of phone numbers targeted by the mass text message, we could not confirm the number of people who actually received the message. Certainly not all people on the list received the message, as a result of insufficient credit purchased for their phone, incorrect profile settings, an invalidly recorded number, no operator coverage, and other possible causes. During an audit of randomly selected 1000 mass text message recipients (544 of whom answered our phone call), $23 \%$ confirmed receiving the message, with no difference between phone numbers assigned to the treatment or placebo film $(p=0.9)$.

\section{Endline survey}

Approximately 10 days after the mass text message was sent to a given community, the endline survey was conducted, from 18 February 2014 to 11 May 2014. Before arriving in each community, surveyors called participants who received a copy of Water of Gold. We collected the phone numbers of friends to add them to the list of mass text message recipients, but only interviewed film buyers in the endline survey (with the exception of one community, where we were unable to contact a sufficient number of film buyers and so interviewed customers and others who had received and watched the film). Surveyors made three attempts to contact each film buyer on the list before moving on to the next person. When surveyors made contact over the phone, they screened the respondent to confirm that they were 18 years of age, which was required for survey participation, and to see whether they had actually watched the film (determined by asking for a cursory description of the plot). Only those who had watched Water of Gold were included in the endline survey. In $91 \%$ of all communities, we interviewed between 12 and 20 people in the community; because of rigorous quality checks, eight communities retained fewer than 12 participants (a minimum of 7). The number of surveys completed is balanced across treatment and placebo (average $=14$ completed surveys each). The endline survey repeated all of the questions contained in the baseline.

\section{Coding incoming messages}

After eliminating incomplete or nonsense texts received by the platform, we coded all remaining messages. Five members of the research team independently coded a random sample of 200 messages and then discussed and agreed on their different interpretations of the coding definitions. One coder then coded all messages using the consensus interpretations, and a second coder coded a random subset of 200 messages, achieving a rate of agreement of $90.5 \%$ (Fleiss' $\kappa=0.81$ ). Messages received between 13 January 2014 and 12 August 2014 were coded and included in the analysis. We report the two main codes of interest in this paper: (i) Any Engagement (texts in which the sender mentioned corruption or the two media campaigns) and (ii) Corruption Report (a subset of Any Engagement messages in which the sender made explicit mention of a corrupt act, person, or institution). A list of the coding definitions with example messages is presented in table S1. While it is impossible to verify the veracity of the reports or to determine the motivations of the sender, we note that most of the messages featured commentary about corruption as a societal problem rather than reports on the malfeasance of specific people. Therefore, the vast majority of messages do not appear to be motivated by a desire for personal vengeance or gain, but rather by a desire to engage with a societal problem.

\section{Analysis}

We predicted the number of corruption messages per day in each community (separately for messages with any engagement and corruption 
report) received from unique senders using the following randomly assigned independent variables: treatment versus placebo film, which was randomly assigned at the geographic level; and day of mass text message reception, which was assigned at the geographic level on a randomly chosen day following film distribution. For the corruption messages, we were able to determine each unique sender's treatment status based on their geographical location, according to the phone company. In all analyses, we interact the film and mass text treatments and used fixed effects for the community matched pair, and we report cluster-robust SEs at the level of the community.

$Y_{i t}=$ treat_film $_{i t}+$ mass_text $_{i t}+$ treat_film $_{i t} \times$ mass_text $_{i t}+$ pair $_{i}$

We predict endline survey responses about social norms of corruption and corruption reporting in each community using the randomly assigned treatment versus placebo film variable. These regressions also control for community matched-pair fixed effects and calculate clusterrobust SEs at the level of community.

$$
Y_{i t}=\text { treat_film } i t+\text { pair }_{i}
$$

\section{SUPPLEMENTARY MATERIALS}

Supplementary material for this article is available at http://advances.sciencemag.org/cgi/ content/full/5/3/eaau5175/DC1

Supplementary Text

Fig. S1. Illustrative map of 106 sampled communities and mobile phone towers.

Fig. S2. Illustrative map of sampled communities in an urban area, Port Harcourt.

Fig. S3. Ex ante predictions for rates of corruption reporting per treatment condition.

Fig. S4. Difference in perceptions of social norms regarding corruption and corruption reporting.

Fig. S5. Difference in perceptions of social norms regarding corruption and corruption reporting, for police and bureaucrat corruption and government corruption.

Table S1. Summary of text messages received in response to the two study campaigns. Table S2. Corruption messages sent as a result of the treatment film and mass text. Table S3. Changes in social norms outcomes as a result of the treatment film. Table S4. Summary statistics for items in social norms outcome indices.

Table S5. Full text of survey questions and response scales used in the design and analysis. Table S6. Corruption messages sent as a result of the treatment film and mass text, averaged over the period before the mass text and the period after.

Table S7. Corruption messages sent as a result of the treatment film and mass text, dropping communities with no film watchers.

Table S8. Corruption messages sent as a result of the treatment film and mass text, dropping communities that received the mass text early.

Table S9. Corruption messages sent as a result of the treatment film and mass text, using assigned mass text send date.

Table S10. Corruption messages sent as a result of the treatment film and mass text, controlling for week of film distribution.

Table S11. Corruption messages sent as a result of the treatment film and mass text interaction, with distribution duration control.

Table S12. Messages related to corruption that are not detailed corruption reports sent as a result of the treatment film and mass text.

Table S13. Changes in social norms outcomes as a result of the treatment film, controlling for individual-level covariates.

Table S14. Changes in social norms outcomes as a result of the treatment film, controlling for week of film distribution start.

Table S15. Changes in social norms outcomes as a result of the treatment film, controlling for the duration of film distribution.

Table S16. Changes in social norms outcomes as a result of the treatment film, with enumerator fixed effects.

\section{REFERENCES AND NOTES}

1. R. Boyd, P. J. Richerson, Punishment allows the evolution of cooperation (or anything else) in sizable groups. Ethol. Sociobiol. 13, 171-195 (1992).

2. M. A. Nowak, K. Sigmund, Evolution of indirect reciprocity. Nature 437, 1291-1298 (2005)

3. R. Boyd, H. Gintis, S. Bowles, Coordinated punishment of defectors sustains cooperation and can proliferate when rare. Science 328, 617-620 (2010).
4. A. Bear, D. G. Rand, Intuition, deliberation, and the evolution of cooperation. Proc. Natl. Acad. Sci. U.S.A. 113, 936-941 (2016).

5. H. Allcott, Social norms and energy conservation. J. Public Econ. 95, 1082-1095 (2011).

6. E. Ostrom, Collective action and the evolution of social norms. J. Econ. Perspect. 14, 137-158 (2000).

7. S. Eidelman, C. S. Crandall, Bias in favor of the status quo. Soc. Personal. Psychol. Compass 6, 270-281 (2012).

8. D. Kahneman, J. L. Knetsch, R. H. Thaler, Anomalies: The endowment effect, loss aversion, and status quo bias. J. Econ. Perspect. 5, 193-206 (1991).

9. E. Fehr, S. Gächter, Altruistic punishment in humans. Nature 415, 137-140 (2002).

10. R. Axelrod, W. D. Hamilton, The evolution of cooperation. Science 211, 1390-1396 (1981).

11. D. Ryvkin, D. Serra, J. Tremewan, I paid a bribe: An experiment on information sharing and extortionary corruption. Eur. Econ. Rev. 94, 1-22 (2017).

12. R. I. Rotberg, J. C. Aker, Mobile phones: Uplifting weak and failed states. Wash. Q. 36 111-125 (2013).

13. M. Zook, M. Graham, T. Shelton, S. Gorman, Volunteered geographic information and crowdsourcing disaster relief: A case study of the haitian earthquake. World Med. Health Policy 2, 7-33 (2010).

14. T. C. Schelling, Dynamic models of segregation. J. Math. Sociol. 1, 143-186 (1971).

15. A. V. Banerjee, A simple model of herd behavior. Q. J. Econ. 107, 797-817 (1992).

16. R. B. Cialdini, M. R. Trost, in The Handbook of Social Psychology, D. T. Gilbert, S. T. Fiske, G. Lindzey, Eds. (McGraw-Hill, ed. 4, 1998), vol. 1-2, pp. 151-192.

17. M. Sherif, The Psychology of Social Norms (Harper, 1936).

18. D. T. Miller, D. A. Prentice, in Social Psychology: Handbook of Basic Principles, E. T. Higgins, A. Kruglanski, Eds. (Guilford Press, 1996), pp. 799-829.

19. M. E. Tankard, E. L. Paluck, Norm perception as a vehicle for social change. Soc. Issues Policy Rev. 10, 181-211 (2016).

20. E. L. Paluck, H. Shepherd, P. M. Aronow, Changing climates of conflict: A social network experiment in 56 schools. Proc. Natl. Acad. Sci. U.S.A. 113, 566-571 (2016).

21. E. La Ferrara, Mass media and social change: Can we use television to fight poverty? J. Eur. Econ. Assoc. 14, 791-827 (2016).

22. A. Banerjee, A. G. Chandrasekhar, E. Duflo, M. O. Jackson, The diffusion of microfinance. Science 341, 1236498 (2013).

23. M. A. Hogg, Influence and leadership, in Handbook of Social Psychology, S. T. Fiske, D. T. Gilbert, G. Lindzey, Eds., (John Wiley \& Sons Inc., Hoboken, NJ, US, 2010), pp. 1166-1207.

24. J. E. Dannals, D. T. Miller, Social norm perception in groups with outliers. J. Exp. Psychol. Gen. 146, 1342-1359 (2017).

25. E. L. Paluck, D. P. Green, Deference, dissent, and dispute resolution: An experimental intervention using mass media to change norms and behavior in Rwanda. Am. Polit. Sci. Rev. 103, 622-644 (2009).

26. R. H. Thaler, C. R. Sunstein, Nudge: Improving Decisions about Health, Wealth, and Happiness (Yale Univ. Press, 2008).

27. S. Bhargava, D. Manoli, Psychological frictions and the incomplete take-up of social benefits: Evidence from an IRS field experiment. Am. Econ. Rev. 105, 3489-3529 (2015).

28. G. B. Chapman, M. Li, H. Colby, H. Yoon, Opting in vs opting out of influenza vaccination. Jama 304, 43-44 (2010).

29. B. Cooke, B. Z. Diop, A. Fishbane, J. Hayes, A. Ouss, A. Shah, Using Behavioral Science to Improve Criminal Justice Outcomes (University of Chicago Crime Lab Report, 2018).

30. B. L. Castleman, L. C. Page, Summer nudging: Can personalized text messages and peer mentor outreach increase college going among low-income high school graduates? J. Econ. Behav. Organ. 115, 144-160 (2015).

31. N. Malhotra, M. R. Michelson, T. Rogers, A. A. Valenzuela, Text messages as mobilization tools: The conditional effect of habitual voting and election salience. Am. Politics Res. 39, 664-681 (2011).

32. D. J. Smith, A Culture of Corruption: Everyday Deception and Popular Discontent in Nigeria (Princeton Univ. Press, 2010).

33. I. Nigeria, egunjedotinfo: Name \& praise or name \& shame...just tell your story!, [accessed 1 September 2016].

34. BribeNigeria.com, Bribenigeria.com: Fighting corruption in nigeria, [accessed 1 September 2016]

35. NOIPolls, Democracy day poll, [accessed 6 September 2016].

36. G. Grossman, M. Humphreys, G. Sacramone-Lutz, Information Technology and Political Engagement: Mixed Evidence from Uganda (Mimeo, 2016).

37. G. Grossman, M. Humphreys, G. Sacramone-Lutz, "I wld like u WMP to extend electricity 2 our village": On Information technology and interest articulation. Am. Polit. Sci. Rev. 108, 688-705 (2014)

38. A. Banerjee, E. Duflo, S. Barnhardt, Movies, margins, and marketing: Encouraging the adoption of iron-fortified salt, in Insights in the Economics of Aging (University of Chicago Press, 2015), pp. 285-306. 
39. M. van Zomeren, R. Spears, A. H. Fischer, C. W. Leach, Put your money where your mouth is! explaining collective action tendencies through group-based anger and group efficacy. J. Pers. Soc. Psychol. 87, 649-664 (2004).

Acknowledgments: We thank R. Gomila for the extraordinary research assistance. We are also very grateful to A. Bland, M. Clark-Barol, A. Coppock, S. Cuerda, J. Drost-Lopez, A. Edgell, M. Gleave, J. Okeke, and M. Offer-Westort for additional research assistance. We thank our partners in the interventions and data collection, especially Integrity Nigeria; iROKO TV; Magic Movies; a major mobile phone firm in Nigeria that wishes to remain anonymous; TNS RMS Nigeria, and especially M. Fagbemi and C. Egbulefu; MK\&l, and especially Y. Aderoju and A. Seosan; Funmobile, and especially A. Adetule; and Social Action. We also thank K. Egbon and K. Henshaw for assistance in developing the interventions, and C. Okpowhor and C. Uche for help in developing and translating our measurement. We are grateful for the useful comments from audiences at Yale, Harvard, Evidence in Governance and Politics (EGAP), the Contemporary African Political Economy Research Seminar (CAPERS), the Paluck laboratory, the UCLA Improving Designs in the Social Sciences workshop, and the American Political Science Association Annual Meeting. Funding: Funding from an anonymous private donor and the Canadian Institute for Advanced Research to E.L.P. is gratefully acknowledged. Ethics statement: This study was approved by the Princeton University Institutional Review Board (protocol no. 5813). The authors complied with all relevant ethical regulations. Author contributions: G.B., R.L., and E.L.P. designed the study, oversaw data collection, analyzed the data, and drafted the manuscript. All authors contributed equally. Competing interests: The authors declare that they have no competing interests. Data and materials availability: All data needed to evaluate the conclusions in the paper are present in the paper and/or the Supplementary Materials. The datasets generated and analyzed during the current study and all codes are available in the OSF repository at http://dx.doi.org/10.17605/OSF.IO/9A7H5.

The experimental design was preregistered at http://egap.org/registration/629.

Submitted 19 June 2018

Accepted 28 January 2019

Published 13 March 2019

10.1126/sciadv.aau5175

Citation: G. Blair, R. Littman, E. L. Paluck, Motivating the adoption of new community-minded behaviors: An empirical test in Nigeria. Sci. Adv. 5, eaau5175 (2019). 